Repère

\title{
Le système éducatif de Hong Kong
}

\section{Béatrice Cabau-Lampa}

\section{CpenEdition}

\section{Journals}

Édition électronique

URL : http://journals.openedition.org/ries/1168

DOI : 10.4000/ries. 1168

ISSN : 2261-4265

Éditeur

Centre international d'études pédagogiques

Édition imprimée

Date de publication : 1 avril 2006

Pagination : 115-121

ISSN : 1254-4590

Référence électronique

Béatrice Cabau-Lampa, "Le système éducatif de Hong Kong ", Revue internationale d'éducation de Sèvres [En ligne], 41 | avril 2006, mis en ligne le 16 novembre 2011, consulté le 01 mai 2019. URL http://journals.openedition.org/ries/1168; DOI : 10.4000/ries.1168

Ce document a été généré automatiquement le 1 mai 2019.

(c) Tous droits réservés 


\title{
Repère \\ Le système éducatif de Hong Kong
}

\author{
Béatrice Cabau-Lampa
}

1 Après le transfert de souveraineté opéré en 1997, la colonie britannique est devenue région administrative spéciale (RAS) de la République populaire de Chine (RPC). L'article 136 de la Loi fondamentale stipule que la RAS - dont près de $95 \%$ de la population est chinoise - définit ses propres politiques en matière de développement et d'amélioration de l'enseignement, y compris les politiques concernant le système éducatif et son administration, la langue dans laquelle l'enseignement est dispensé, les crédits, le système d'examens, le système des titres universitaires et la reconnaissance des diplômes. Selon l'article 137, les établissements d'enseignement peuvent conserver leur autonomie et jouir de la liberté d'enseignement et les élèves sont libres de choisir leur établissement ${ }^{1}$. En 2004/2005, on comptait 737 écoles maternelles (qui fonctionnent la plupart du temps seulement en demi-journée), 760 écoles primaires, 519 écoles secondaires et 66 écoles à pédagogie spéciale (pour élèves malvoyants, malentendants...).

\section{8 : un premier train de réformes}

Depuis 1978, le système scolaire comprend neuf années de scolarité obligatoire et gratuite : six années de primaire suivies de trois années de secondaire du premier cycle pour les jeunes de six à quinze ans. L'enseignement secondaire supérieur est payant ${ }^{2}$. Il consiste en quatre classes de deux années chacune : les deux premières ( $\mathrm{S} 4$ et $\mathrm{S} 5$ ) mènent au Hong Kong Certificate of Education (HKCEE), les deux suivantes (S6 et S7) au Hong Kong Advanced Level Examination (HKALE) pour les élèves ayant obtenu de bons résultats en S7. Depuis 2004, les élèves ne pouvant ou ne désirant pas terminer leurs études secondaires et obtenir le HKCEE ont la possibilité de suivre un programme spécial organisé par le Bureau de l'éducation et de l'emploi dans plusieurs écoles secondaires. $\mathrm{Si}$, au terme du programme, leurs résultats sont satisfaisants, ils se voient décerner un certificat reconnu dans le cadre de la formation continue et de la recherche d'emploi.

3 La même année, une ordonnance scolaire a relevé les critères d'admission pour les enseignants des jardins d'enfants, du primaire et du secondaire. C'est ainsi qu'il n'est plus 
possible pour ceux qui possèdent une longue expérience professionnelle d'être exemptés de formation professionnelle. Une convention de reconnaissance mutuelle des diplômes universitaires entre la RPC et la RAS a été signée en 1978 également.

\section{L'accès à l'enseignement supérieur : une réforme à venir}

Ce sont les résultats scolaires qui déterminent l'accès à l'enseignement supérieur, lequel comprend douze institutions, dont huit subventionnées par le Comité pour l'octroi de subventions aux universités (University Grants Committee), sept d'entre elles étant des universités. Le pourcentage des jeunes suivant des études dans une de ces institutions est passé de 6-7 \% à la fin des années 1980 à $18 \%$ de nos jours. À ces chiffres s'ajoutent ceux de la formation professionnelle et des programmes ne menant pas à l'obtention de la licence ou encore ceux des étudiants partant à l'étranger (en Australie, en GrandeBretagne, aux États-Unis, le Canada ou la Nouvelle-Zélande) pour poursuivre leurs études, soit $39 \%$. Le taux total de participation au niveau post-secondaire des jeunes âgés de dixsept à vingt ans est passé d'environ $30 \%$ en 2000/2001 à $57 \%$ en 2004/2005 ${ }^{3}$. Par ailleurs, pour faire de Hong Kong un centre régional d'éducation et attirer des étudiants non locaux, les mesures de contrôle d'immigration sont assouplies depuis l'année universitaire 2005/2006.

Néanmoins, le système n'arrive pas à produire assez de diplômés : en 1999/2000, 55 \% des élèves quittaient l'école à la fin de l'enseignement secondaire du premier cycle et seulement $25 \%$ des écoliers du secondaire accédaient aux études supérieures contre une moyenne d'environ $40 \%$ dans les pays de l'OCDE ${ }^{4}$. La situation ne s'est pas améliorée récemment : à l'heure actuelle, près des deux tiers des élèves ne passent pas en S6. C'est pourquoi en 2000, Tung Chee-Hwa, alors chef de l'exécutif de la RAS, a déclaré qu'en 2010/2011, 60 \% des jeunes âgés de dix-sept à vingt ans devaient accéder à l'enseignement supérieur. En 2009 sera appliquée une réforme de l'enseignement secondaire supérieur et de l'enseignement supérieur selon le schéma 3+3+4. La scolarité des jeunes Hongkongais se déroulera comme suit : trois années dans le premier cycle du secondaire (classes $\mathrm{S} 1$ à $\mathrm{S}^{3}$ ) suivies de trois autres au lycée ( $\mathrm{S}^{4}$ à $\mathrm{S6}$ ), puis de quatre années dans un établissement d'enseignement supérieur pour obtenir une licence (et non plus trois, comme à présent). Tous les écoliers suivront donc une scolarité de six années dans le secondaire et la classe S7 deviendra partie intégrante de l'enseignement supérieur. Les deux examens - le HKCEE ainsi que le HKALE - seront supprimés et remplacés en 2011/2012 par un nouveau système d'évaluation plus globale pour l'obtention d'un unique diplôme, le Hong Kong Diploma of Secondary Education. Le système existant est considéré comme orienté vers la course aux examens et la réforme supposée augmenter le nombre d'écoliers poursuivant l'intégralité de la scolarité du secondaire et réduire ainsi le taux d'abandons. Le tableau ci-contre présente le contenu du nouveau programme au niveau du lycée (New Senior Secondary).

\begin{tabular}{|l|l|l|}
\hline Programme & Niveau lycée & $\begin{array}{l}\text { Pourcentage en } \\
\text { temps } \\
\text { d'enseignement }\end{array}$ \\
\hline
\end{tabular}




\begin{tabular}{|l|l|l|}
\hline Matières principales & $\begin{array}{l}\text { Chinois*, anglais, mathématiques et cours de } \\
\text { culture générale }\end{array}$ & $45-55 \%$ \\
\hline $\begin{array}{l}\text { Matières } \\
\text { optionnelles }\end{array}$ & $\begin{array}{l}\text { Deux ou trois choisies parmi vingt matières du } \\
\text { nouveau programme au niveau lycée et un choix } \\
\text { de matières à orientation professionnelle }\end{array}$ & $20-30 \%$ \\
\hline $\begin{array}{l}\text { Autres expériences } \\
\text { d'apprentissage }\end{array}$ & $\begin{array}{l}\text { Éducation civique et morale, service pour la } \\
\text { commuanuté, activités esthétiques et civiques, } \\
\text { expériences liées à la perspectives de carrière } \\
\text { professionnelle }\end{array}$ & $15-35 \%$ \\
\hline
\end{tabular}

* Des modules à option de mandarin seront proposés aux écoliers désireux d'approfondir leurs connaissances dans cette matière. Voir note de bas de page $n^{\circ} 5$.

\section{Complexité des infrastructures d'accueil}

6 Le secteur public comprend les governement schools, établissements entièrement gérés par le Education and Manpower Bureau (une minorité) et les écoles gérées par diverses associations souvent religieuses mais entièrement subventionnées par le gouvernement, et dont la présence remonte aux premières années de la Colonie. Ces deux types d'établissements reçoivent une subvention du gouvernement basée sur le nombre de classes (à raison de trente-sept élèves par classe de primaire et quarante dans le secondaire).

7 Ce sont les écoles DSS (Direct Subsidy Scheme, programme de subventions directes introduit en 1991) qui profitent le plus de l'expansion du secteur non public et plusieurs écoles privées ont rejoint le DSS afin de pouvoir bénéficier des subventions du gouvernement. Si ces écoles reçoivent la même subvention par écolier que les écoles publiques, elles ont le droit de demander des frais de scolarité (de 10000 à $50000 \mathrm{HK} \$$ ). Elles jouissent d'une grande liberté en matière d'administration, de sélection et de programme ainsi que d'une image d'écoles d'élite, ce qui les rend très attrayantes aux yeux des parents. À la différence des écoles privées indépendantes, elles doivent offrir un programme scolaire menant aux examens locaux.

8 Pour offrir une éducation de style britannique aux enfants de fonctionnaires et hommes d'affaires britanniques, la Fondation des écoles anglaises (English Schools Foundation), créée en 1967, constitue la principale source d'éducation internationale avec dix-neuf établissements, dont dix écoles primaires et cinq écoles secondaires. Le rôle qu'elle a joué durant l'ère coloniale lui a permis de recevoir une subvention importante (même si celleci a subi plusieurs réductions) bien que moins élevée que celle des écoles DSS. Elle a pu, ainsi, demander des frais d'admission inférieurs à ceux des écoles internationales (les frais annuels vont de 47300 à $78600 \mathrm{HK} \$$ pour le primaire et le secondaire), tout en offrant à ses enseignants des salaires parmi les plus élevés au monde (même s'il est question de les réduire de $9 \%$ ). Certains s'interrogent sur le bien-fondé de l'existence d'écoles offrant un enseignement de style britannique en période post-coloniale, subventionnées par les contribuables hongkongais, alors qu'une grande partie des élèves n'est pas chinoise et est issue de familles aisées. 
9 Les cinquante-six écoles internationales, subventionnées pour la plupart par leur pays d'origine, répondent à une forte et croissante demande de parents expatriés mais aussi hong kongais malgré leurs tarifs très élevés (pouvant dépasser les $130000 \mathrm{HK}$ ). Elles peuvent offrir un programme américain, australien, britannique, australien, mais aussi suisse-allemand, singapourien, japonais, coréen, français (Lycée français International Victor Ségalen)... aboutissant au baccalauréat international.

Enfin, il existe huit écoles privées indépendantes (Private Independent Schools), créées à la fin des années 1990 pour offrir une alternative locale aux meilleures écoles internationales. Indépendantes financièrement et couvrant leurs dépenses par les frais d'admission, elles sont libres de choisir leur programme scolaire.

\section{Quelle langue d'enseignement ? Le cantonais, le mandarin ou l'anglais?}

11 Jusqu'en 1990, la langue d'enseignement n'était déterminée par aucun texte officiel même si, depuis les années 1970, plusieurs rapports sur l'éducation se sont prononcés en faveur de l'enseignement en langue maternelle, à savoir le cantonais ${ }^{5}$ et le chinois a été décrété langue officielle en 1974 sur un pied d'égalité avec l'anglais. Selon une politique de «laisser-faire », les établissements scolaires avaient le droit de dispenser leur enseignement soit en anglais, soit en chinois. Il faut dire qu'avant 1978, année de la création de l'école obligatoire d'une scolarité de neuf ans, cette question ne concernait encore qu'un petit groupe d'élites. Dans les années 1980, elle est devenue l'objet de nombreux débats non seulement dans le milieu éducatif mais aussi au sein de la communauté. Il est également à signaler qu'avant 1978 comme de nos jours, le chinois est utilisé dans le cycle primaire, pour être généralement remplacé par l'anglais dans le secondaire, sauf pour une minorité d'élèves qui poursuivent leurs études dans des établissements où est utilisé le chinois. La différence est qu'à cette époque, l'anglais était véritablement la langue d'enseignement dans la majorité des écoles. L'éducation de masse a entraîné une baisse du niveau d'anglais dans la population étudiante et les enseignants, ce qui a rendu l'enseignement dans cette langue difficile, voire impossible dans plusieurs écoles. En fait, depuis 1978, très peu d'établissements dispensent un enseignement uniquement en chinois ou en anglais. L'enseignement se déroule sur un mode mixte ( mixed-code), dans un mélange d'anglais et de chinois ${ }^{5}$.

Dans la perspective de remédier à ces problèmes, les membres de la Commission sur l'éducation de 1990 ont rédigé leur rapport, le premier du genre en matière de politique linguistique dans le domaine scolaire, en préconisant les principes suivants ${ }^{6}$ :

- les établissements d'enseignement secondaire devront être encouragés à adopter le chinois comme langue d'enseignement, ce qui devrait rendre, en règle générale, l'enseignement/apprentissage plus efficace ;

- la décision d'opter pour le chinois ou l'anglais en tant que langue d'instruction revient à la direction de chaque établissement scolaire ;

- l'utilisation du mode mixte devra être réduite en faveur de l'utilisation dans chaque cours, de façon clairement définie, du chinois ou de l'anglais, selon l'enseignement en question, les manuels utilisés et les examens préparés.

13 Ce texte de 1990 reflète en fait une volonté de compromis et de flexibilité, afin de satisfaire l'ensemble de la population. Dès le 3 juillet 1997, Tung Chee-Hwa déclarait que 
le gouvernement de Hong Kong se devait de continuer ses efforts pour améliorer l'enseignement des langues, l'objectif étant de former des étudiants capables de maîtriser l'anglais et le chinois à l'écrit et de parler couramment l'anglais, le mandarin et le cantonais. En avril de la même année, le ministère de l'Éducation adressait à toutes les écoles un document intitulé «Arrangements for Firm Guidance on Secondary Schools' Medium of Instruction». Une réorientation de la politique linguistique dans le cadre scolaire décrétait que le chinois était la seule langue d'enseignement, sauf pour les cours d'anglais et de littérature anglaise. Il s'en suivit une levée de boucliers qui amena le ministère à se rétracter et à publier une version révisée de ce texte en septembre, "the Medium of Instruction Guidance for Secondary Schools». Comme l'indique le titre, le ton du second document est moins directif, même si l'objectif reste le même : l'enseignement en langue maternelle et la suppression des établissements dispensant un enseignement en chinois et en anglais (two medium schools). Le type particulier d'éducation bilingue en vigueur avant 1978 - chinois dans le primaire, anglais dans le secondaire - est donc rejeté au profit de l'enseignement unilingue, dans la langue maternelle. La flexibilité offerte dans le rapport de la Commission de 1990 est supprimée. Lorsque le ministère publia, en décembre 1997, la liste des cent établissements ayant le droit d'utiliser l'anglais comme langue d'enseignement (environ $25 \%$ du total des écoles secondaires) sauf pour les cours de langue et d'histoire chinoises, vingt écoles firent appel et quatorze d'entre elles eurent gain de cause ; la plupart des écoles secondaires craignent de ne plus attirer «les bons élèves " en adoptant la langue maternelle comme outil d'enseignement. Néanmoins, le rapport de la Commission de l'éducation a réitéré en 2005 le principe de la langue maternelle comme langue d'enseignement dans les écoles secondaires, conjugué à l'objectif d'amélioration de l'enseignement de l'anglais ?

14 Le désir d'instituer le chinois comme langue d'enseignement est à relier avec un autre objectif éducatif qui découle du transfert de souveraineté. Le passage de l'état de colonie à celui de région administrative spéciale de la RPC a engendré le besoin de valoriser l'éducation civique afin que les jeunes Hongkongais comprennent mieux la Chine, sa culture, son histoire, le concept « un pays, deux systèmes » et la constitution de Hong Kong. Tung Chee-Hwa espérait qu'ainsi, le système éducatif de Hong Kong inculquerait aux élèves une passion et un intérêt pour la Chine, la fierté d'être chinois, ainsi qu'une volonté sans faille de contribuer au bien-être de Hong Kong mais également de tout le pays. Il serait en effet politiquement difficile pour la RAS de dédaigner le mandarin en favorisant l'anglais.

15 Cependant, l'anglais reste indispensable pour le développement économique de Hong Kong, centre cosmopolite des affaires et de la finance. Il n'est plus perçu comme la langue coloniale, mais comme un outil indispensable de communication. Diverses voix se sont élevées pour déplorer la baisse du niveau dans cette langue : employeurs, représentants d'entreprises commerciales étrangères, touristes, etc., sans oublier la pression exercée par l'entrée de la Chine dans l'Organisation mondiale du Commerce en 2001. Afin d'y remédier, le gouvernement a introduit en 2001 un test d'évaluation de performance pour les professeurs d'anglais dans l'enseignement secondaire, sauf pour ceux possédant un diplôme universitaire d'anglais et ayant suivi une formation professionnelle. Selon les enseignants, opposés à cette réforme qu'ils jugent insultante, ce ne sont pas leurs compétences qui doivent être remises en question, mais plutôt la pénurie d'enseignants d'anglais : certains établissements scolaires demandent à leurs professeurs d'enseigner l'anglais langue, alors qu'ils ne possèdent aucune qualification dans cette matière. Les 
enseignants d'anglais ont également mis en cause le nombre élevé d'élèves par classe ainsi que la stratégie du système éducatif. Le problème du recrutement d'enseignants d'anglais qualifiés est loin d'être résolu : en 2003, près de $70 \%$ des 1930 enseignants qui ont passé le test ont échoué à l'écrit ${ }^{8}$ et la situation ne s'est pas améliorée l'année suivante.

\section{Quelle école?}

Le deuxième problème majeur des réformes du système éducatif est le système d'affectation des places dans les écoles secondaires. Jusqu'en 2000, le test d'aptitude scolaire (Academic Aptitude Test) que devaient passer les élèves de Primary Six (P6), déterminait l'évaluation des écoliers et leur affectation dans un établissement secondaire. Les élèves «bûchaient » des mois, voire des années, afin d'être admis dans les meilleures écoles. Depuis 2000, les élèves ont été regroupés par tranches d'aptitude selon leurs résultats scolaires et en 2001, le nombre de tranches (band) a été réduit de cinq à trois afin d'augmenter l'éventail d'aptitudes des élèves dans les écoles et de faire disparaître les stigmates de l'échec (la classification en dernière tranche), la principale critique étant que le système à cinq tranches était trop élitiste. Toujours est-il que la sélection s'effectue toujours à l'âge de onze ans sur la base des résultats scolaires qui déterminent les chances d'entrer dans les meilleures écoles secondaires : le tiers du nombre total des élèves ayant obtenu les meilleures notes aux examens de P5 et P6 et autres devoirs (comptant respectivement pour 80 et $20 \%$ de l'évaluation totale) sont classés dans la tranche 1 (il s'agit des filles en majorité) et ainsi sélectionnés par les meilleures écoles secondaires. Ce système, fort critiqué par les pédagogues et chercheurs, ne sera pas abandonné dans un proche avenir car les parents ne sont pas prêts à y renoncer et les écoles rechignent à affronter l'hétérogénéité au sein du public scolaire 9.

Les actuels débats sur la réforme de l'enseignement exposent la difficulté de combiner plusieurs enjeux : la construction d'une nouvelle identité nationale avec la nécessité de préserver une certaine philosophie éducative (dans laquelle l'élitisme et l'investissement privé sont encouragés) héritée du système britannique et chère à une grande partie du public hongkongais, sans oublier les enjeux économiques et l'impact de la globalisation. On peut se demander si les jeunes vont s'y retrouver. D'ailleurs, si l'enquête PISA 2003 a révélé les excellents résultats des élèves hongkongais en culture mathématique, sciences et résolution de problèmes, elle a également fait apparaître que c'est la population scolaire de Hong Kong qui manifeste l'attitude la plus négative à l'égard de l'école.

\section{NOTES}

1. Haut commissariat des Nations unies aux droits de l'homme de Genève : http://www.hri.ca/ fortherecord2001/bilan2001/documentation/tbodies/e-1990-5-add43.htm

2. En 2005 , les frais annuels s'élevaient à $5050 \mathrm{HK} \$$ pour les classes $\mathrm{S} 4$ et $\mathrm{S} 5$, et à 8750 pour les classes S6 et S7 ( $1 \mathrm{HK} \$=0,10$ euro). 
3. Hong Kong Special Administrative Region Government (2005), Hong Kong Yearbook, 2004.

4. Strategy Policy and Research in Education Ltd, Four reforms - Hong Kong's Demand for International Education, April 2001.

5. Ce mode mixte pouvait s'opérer soit dans la même phrase, soit sous la forme de traduction systématique en chinois de chaque phrase en anglais, soit encore sous forme de la présentation d'une idée en anglais, suivie d'une explication en chinois, soit, enfin, sous forme d'alternance linguistique par phases: introduction en anglais, développement en chinois et conclusion en chinois.

6. Poon A. «Bilingualism and monolingualism: a shift in Hong Kong's language in education policy ", in Berry V., McNeill A. (1998), Policy and Practice in Language Education, the University of Hong Kong, Department of Curriculum Studies, pp. 89-104.

7. Education Commission, Report on Review of Medium of Instruction for Secondary Schools and Secondary School Places Allocation, December 2005.

8. "Teachers fail to make the grade in language tests ", South China Morning Post, 19 décembre 2003.

9. « Children march to bands of pressure », South China Morning Post, 12 mars 2005.

INDEX

Mots-clés : politique éducative, système éducatif

Index géographique : Hong-Kong

\section{AUTEUR}

\section{BÉATRICE CABAU-LAMPA}

Associate-Professor, European Studies Programme, Coordinator for the French Stream, Hong Kong Baptist University. 